\title{
Development of a maternity hospital classification for use in perinatal research
}

\author{
Michael O. Falster ${ }^{\mathrm{A}, \mathrm{B}}$, Christine L. Roberts ${ }^{\mathrm{A}, \mathrm{E}}$, \\ Jane Ford $^{\mathrm{A}}$, Jonathan Morris ${ }^{\mathrm{A}, \mathrm{C}}$, Ann Kinnear \\ and Michael Nicholl ${ }^{\mathrm{C}, \mathrm{D}}$ \\ ${ }^{\mathrm{A} C l i n i c a l}$ and Population Perinatal Research, Kolling Institute \\ of Medical Research, Royal North Shore Hospital \\ ${ }^{\mathrm{B}}$ NSW Biostatistical Officer Training Program, \\ NSW Department of Health \\ ${ }^{\mathrm{C}}$ Department of Obstetrics, Gynaecology and Neonatology, \\ The University of Sydney at Royal North Shore Hospital \\ ${ }^{\mathrm{D}}$ Primary Health and Community Partnerships, \\ NSW Department of Health \\ ${ }^{\mathrm{E}}$ Corresponding author. Email: christine.roberts@sydney.edu.au
}

pregnancies less than 33 weeks gestation be delivered at hospitals with neonatal intensive care units to ensure babies are born under the best conditions possible to reduce morbidity and mortality of the newborn. ${ }^{5}$

In New South Wales (NSW), information about all births is collected in the NSW Perinatal Data Collection (PDC), a population-based statutory surveillance system which includes information on maternal characteristics, pregnancy, birth and infant outcomes. An obstetric service level is assigned to each hospital where women give birth. These levels are based on a complex array of maternity and neonatal staffing, expertise, pathology and surgical and anaesthetic capability, and range from Level 6 (providing both obstetric and neonatal tertiary care) to Level 1 (no birthing services, may provide postnatal care). ${ }^{6}$ Although the components of the level can change throughout the course of a year (e.g. in rural hospitals the departure of the only obstetrician would change the level), the level that has prevailed for the majority of the year is assigned as the level for the entire year.

When using these levels for research, other limitations emerge. Firstly, there is no geographical differentiation, although the provision and outcome of maternity services needs to take geography into account. Secondly, no service levels are assigned to private hospitals as no information on maternity or neonatal services capability in private hospitals in NSW is routinely available; they form one group regardless of the level of obstetric and neonatal services available. Thirdly, there is no evidence that the level designations are better predictors of birth outcome than the annual number of births at each hospital. ${ }^{7}$ Therefore we aimed to develop a service level descriptor that was suitable for research with stable and easily available components that would have wide application and allow comparison across state, national and international jurisdictions. We wanted groupings of hospitals that were sufficiently large that would allow us to exclude or collapse the groups across dimensions relevant to a research question. Here we report the development of such a classification of maternity hospitals and provide a case study of its use.

\section{Methods - construction of a new maternity hospital classification}

We classified maternity hospitals in NSW according to the following dimensions of service level: neonatal care 
Table 1. Classification of maternity hospitals in NSW by 13 obstetric groups including home births, 2001 and 2008

\begin{tabular}{|c|c|c|c|c|c|}
\hline \multirow[t]{2}{*}{ Hospital obstetric group } & \multicolumn{3}{|c|}{ Grouping criteria } & \multicolumn{2}{|c|}{ Hospitals } \\
\hline & Resources & Geographic area & $\begin{array}{l}\text { Annual birth volume } \\
n\end{array}$ & $\begin{array}{c}2001 \\
n\end{array}$ & $\begin{array}{c}2008 \\
n\end{array}$ \\
\hline $\mathrm{NICU}$ & Tertiary public & Any region & $\geq 1000$ & 7 & 7 \\
\hline CPAP $(2001+)$ & CPAP facilities public & Any region & $\geq 1000$ & 5 & 5 \\
\hline Large urban & Non tertiary public & Urban & $\geq 1000$ & 4 & 6 \\
\hline Medium urban & Non tertiary public & Urban & $500-999$ & 7 & 3 \\
\hline Small urban & Non tertiary public & Urban & $20-499$ & 2 & 3 \\
\hline Large regional & Non tertiary public & Regional & $\geq 1000$ & 3 & 5 \\
\hline Medium regional & Non tertiary public & Regional & $500-999$ & 10 & 8 \\
\hline Small regional & Non tertiary public & Regional & $20-499$ & 48 & 39 \\
\hline Large private & Private hospital & Any region & $\geq 1000$ & 9 & 9 \\
\hline Medium private & Private hospital & Any region & $500-999$ & 6 & 6 \\
\hline Small private & Private hospital & Any region & $20-499$ & 9 & 4 \\
\hline Other/postnatal & Non tertiary public & Any region & $<20$ & 26 & 20 \\
\hline Home births & - & Any region & N/A & N/A & $\mathrm{N} / \mathrm{A}$ \\
\hline $\begin{array}{l}\text { CPAP: continuous positive air } \\
\text { NICU: neonatal intensive care } \\
\text { Source: Population Health Re }\end{array}$ & \multicolumn{4}{|c|}{ CPAP: continuous positive airways pressure. } & \\
\hline
\end{tabular}

capability (tertiary neonatal intensive care unit (NICU); continuous positive airways pressure (CPAP) facilities and trained staff $^{8}$ or other); geography (urban or rural location); annual average number of births ( $>1000,500-999$, $20-499$ and $<20$ births); and hospital status (public or private). Women birthing outside a hospital were classified as a separate group. These criteria were used to create a set of 13 obstetric groups (12 hospital groups plus home births) (Table 1). Although a classification with 13 levels is unwieldy for most research purposes, the hospital groups can easily be collapsed along dimensions that are appropriate to the study objectives. Groupings can be allocated on the basis of a priori research questions and/or similarity of clinical characteristics prior to the assessment of outcomes, as in the following case study. SAS program coding for the maternity hospitals classification is available on the Population Health Research Network website at www.phrn.org.au.

\section{Results - case study}

Two objectives of a recent study of early elective births (induction of labour or pre-labour caesarean section) in NSW were to determine the risk of severe neonatal morbidity following elective births (33-39 weeks gestation), and the extent to which the morbidity differs according to the gestational age at which the infant was born. ${ }^{9}$ While much is known about the pattern of morbidity by gestational age in tertiary hospitals, ${ }^{10}$ less is known about the patterns of, and outcomes following, elective deliveries in a range of non-tertiary hospitals. Thus, a secondary aim of the study was to assess patterns of morbidity across non-tertiary hospitals, where there may not be the service capability to care for sick and preterm infants.

Data on births were obtained from the NSW Perinatal Data Collection. To assess patterns of morbidity, birth data were linked with hospital data in the NSW Admitted Patient Data Collection. Neonatal and maternal outcomes were assessed using composite indicators of morbidity which include both diagnoses and procedures, and are able to overcome problems of under-ascertainment of individual adverse events. ${ }^{11,12}$ For the purposes of this study, home births and hospitals offering postnatal or midwifery-led care were excluded as elective births do not occur in these settings.

Hospitals were initially stratified into the 12 maternity hospital groups. Rates of elective births (by method) were compared across hospital strata and by gestational age, and strata were combined when the pattern of rates were similar across gestational ages. For example, the elective birth rates for the three levels of private hospitals were grouped based on similar rates across gestational ages (Figure 1). This resulted in six hospital groups: tertiary hospitals, hospitals with continuous positive airways pressure facilities, all other urban hospitals, large regional hospitals (delivery volume $\geq 1000$ ), all other regional hospitals (delivery volume $<1000$ ), and private hospitals.

Figure 2 ( $a$ and $b$ ) presents the absolute risk of severe neonatal morbidity by gestational age and hospital classification. There is a stepwise decline in neonatal morbidity 


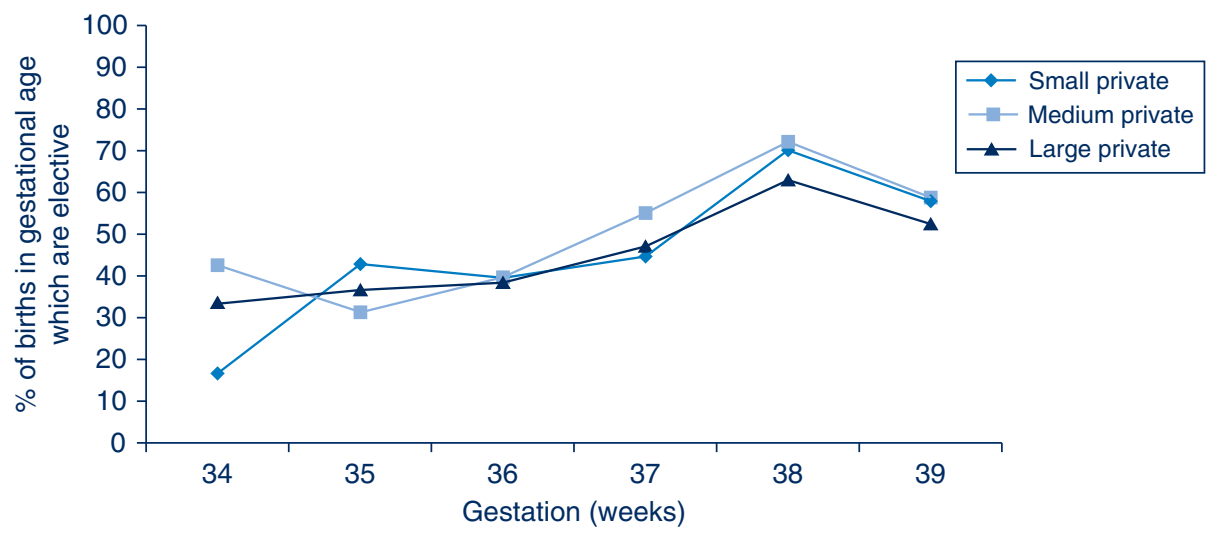

Figure 1. Gestation-specific rates of elective births at private hospitals, NSW, 2001-2007. Source: NSW Perinatal Data Collection.
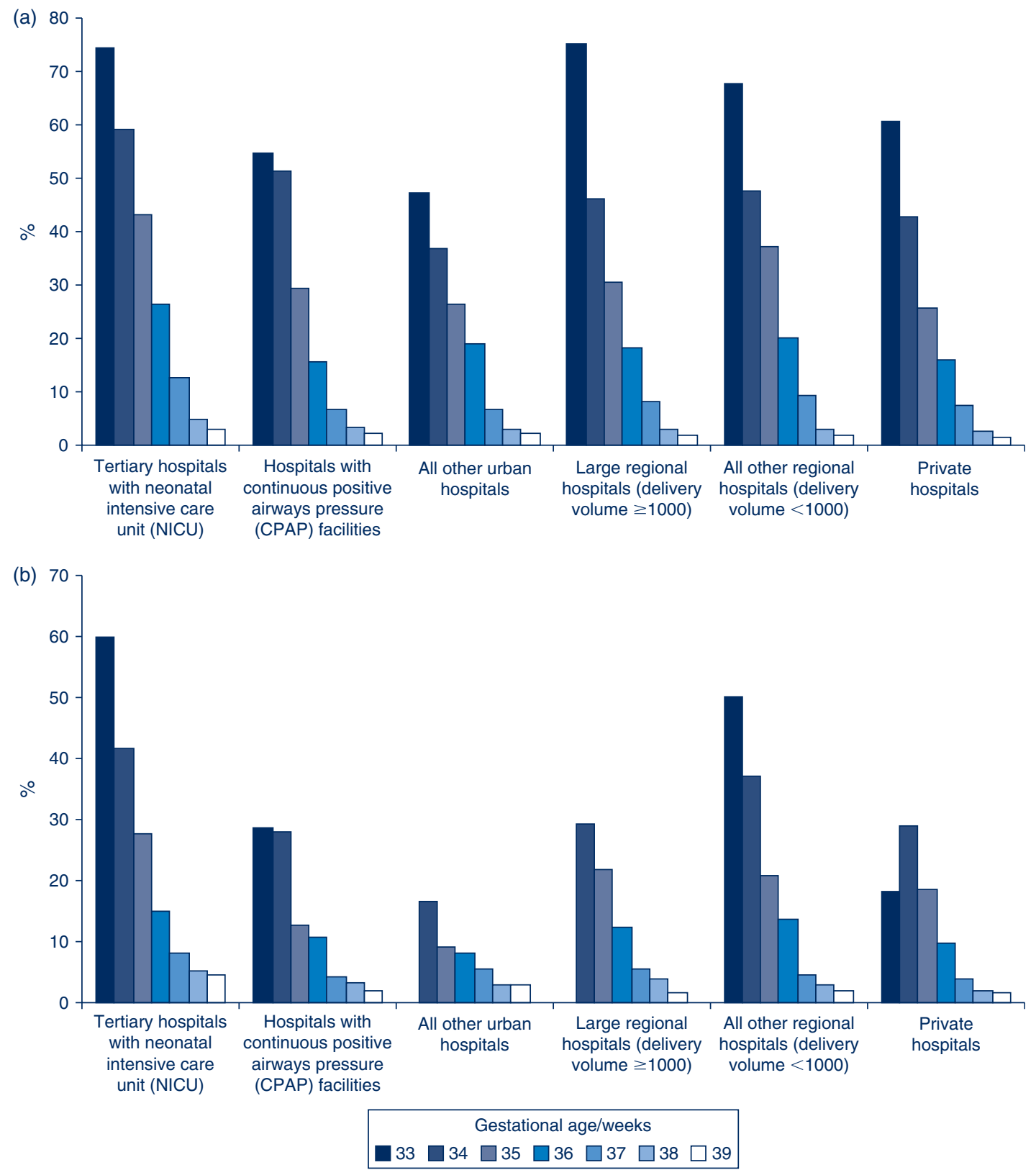

Figure 2. Absolute risk of neonatal morbidity at seven gestational ages following elective birth, by hospital classification, using six maternity hospital groups, NSW, 2001-2007. (a) Following pre-labour caesarean. (b) Following induction.

Source: Linked NSW Perinatal Data Collection and NSW Admitted Patient Data Collection. 
with each week of advancing gestation irrespective of the mode of elective birth, and this stepwise pattern was present in all of the hospital groups. The highest gestation-specific morbidity rates were at hospitals with a neonatal intensive care unit, which is consistent with birth of high-risk infants in tertiary centres. The rates of morbidity were higher following pre-labour caesarean section than induction at every gestation until 39 weeks. The pattern of stepwise improvement in outcomes associated with increasing gestation was also observed for maternal morbidity, and rates of transfer to a neonatal intensive care unit for infants born in non-tertiary hospitals (data not shown).

\section{Discussion}

Classifying hospitals into service levels is important for health services research. However, the classification of hospitals into similar groups may vary depending on the research question. In the case study presented, a key focus of the study was access to neonatal care and therefore geographical and resources dimensions were important.

Our case study found higher rates of severe adverse outcomes at shorter gestations with a stepwise decline as gestation increases, especially following pre-labour caesarean section. This pattern was found across all hospital classifications, including a range of non-tertiary hospitals that may not have the facilities to care for sick and preterm infants, reaffirming the importance of birth in risk-appropriate settings. Such results highlight the need for health policy to address the accessibility of obstetric and neonatal support services. Role delineation guidelines need to incorporate criteria on elective birth (pre-labour caesarean and induction of labour) and resourcing of regional hospitals needs to be reviewed if elective births are to be sanctioned in such settings.

The groupings in the case study are pragmatic from a clinical and policy decision-making perspective, and appear to have validity in the expected pattern of morbidity. The collapsed set of six groups was manageable for analysis, and was easily interpretable in the context of the study purposes. In the case study, rates of elective delivery were more similar between private hospitals, than between private and public hospitals of similar geography and volume, and so private hospitals were grouped together.

A limitation of the hospital groups may be the immediate application to jurisdictions outside of NSW, although the classification is easily adapted. For example, there may be private hospitals with neonatal intensive care unit facilities in other jurisdictions, and so an additional category may be needed. Further identification of specialised services, such as hospitals that now offer midwifery care only, is also possible. The proposed classification increases the potential for comparability, through greater flexibility and transparency in the classification of groups. Furthermore, in the absence of available perinatal data, the groups may be replicated using alternate data sources such as hospitalisation data.

\section{Conclusion}

We have developed and used a classification of maternity hospitals that is based on readily available information, that may be adapted to different research questions and could be used at an area, state, national or international level. Hospitals will change groups if there are significant changes to the annual birth volume, avoiding reliance on facilities and staffing change which are hard to monitor in over 100 hospitals. This approach may be adaptable to other service delivery areas.

\section{Acknowledgments}

We thank the NSW Ministry of Health for access to the population health data and the NSW Centre for Health Record Linkage for linking the data sets. This work was supported by an Australian National Health and Medical Research Council (NHMRC)/NSW Ministry of Health Partnership Grant (\#571451). MOF was employed as part of the NSW Biostatistical Officer Training Program funded by the NSW Ministry of Health while undertaking this work at the Kolling Institute of Medical Research.

\section{References}

1. Keeler EB, Rubenstein LV, Kahn KL, Draper D, Harrison ER, McGinty MJ et al. Hospital characteristics and quality of care. JAMA 1992; 268(13): 1709-14. doi:10.1001/ jama.1992.03490130097037

2. Phibbs CS, Bronstein JM, Buxton E, Phibbs RH. The effects of patient volume and level of care at the hospital of birth on neonatal mortality. JAMA 1996; 276(13): 1054-9. doi:10.1001/ jama.1996.03540130052029

3. Birkmeyer JD, Stukel TA, Siewers AE, Goodney PP, Wennberg DE, Lucas FL. Surgeon volume and operative mortality in the United States. N Engl J Med 2003; 349(22): 2117-27. doi:10.1056/NEJMsa035205

4. Badawi N, Adelson P, Roberts C, Spence K, Laing S, Cass D. Neonatal surgery in New South Wales-what is performed where? J Pediatr Surg 2003; 38(7): 1025-31. doi:10.1016/ S0022-3468(03)00184-2

5. National Health and Medical Research Council. Clinical practice guidelines: care around preterm birth. Canberra: NHMRC; 1997.

6. NSW Health Department. Guide to role delineation of health services. Sydney: NSW Health; 2002.

7. Algert CS, Morris JM, Bowen JR, Giles W, Roberts CL. Twin deliveries and place of birth in NSW 2001-2005. Aust N ZJ Obstet Gynaecol 2009; 49(5): 461-6. doi:10.1111/j.1479828X.2009.01054.X

8. Buckmaster AG, Arnolda G, Wright IM, Foster JP, HendersonSmart DJ. Continuous positive airway pressure therapy for 
infants with respiratory distress in non tertiary care centers: a randomised, controlled trial. Pediatrics 2007; 120(3): 509-18. doi:10.1542/peds.2007-0775

9. Falster MO, Roberts CL, Ford J, Morris J, Nicholl M. Informing hospital role delineation: Elective delivery of pregnant women before the due date. Aust Epidemiol 2010; 17(2): 48.

10. Bailit JL, Gregory KD, Reddy UM, Gonzalez-Quintero VH, Hibbard JU, Ramirez MM et al. Maternal and neonatal outcomes by labor onset type and gestational age. $\mathrm{Am} \mathrm{J}$ Obstet Gynecol 2010; 202(3): 245.e1-e12. doi:10.1016/ j.ajog.2010.01.051
11. Lain S, Algert C, Nassar N, Bowen J, Roberts C. Incidence of Severe Adverse Neonatal Outcomes: Use of a Composite Indicator in a Population Cohort. Matern Child Health $J$ 2011 April. [Epub ahead of print]. doi:10.1007/s10995-0110797-6

12. Roberts CL, Cameron CA, Bell JC, Algert CS, Morris JM. Measuring maternal morbidity in routinely collected health data: development and validation of a maternal morbidity outcome indicator. Med Care 2008; 46(8): 786-94. doi:10.1097/ MLR.0b013e318178eae4 